\title{
Publisher Correction: Tomato multiomics at aPEELing resolution
}

Alisdair R. Fernie (1) and Saleh Alseekh (1)

Correction to: Nature Plants https://doi.org/10.1038/s41477-020-00807-8, published online 3 November 2020.

In this News \& Views originally published, the two central images of tomatoes in Fig. 1 had been used without permission; those images have now been replaced with photos taken by the authors and the figure has been updated in all versions.

Published online: 1 December 2020

https://doi.org/10.1038/s41477-020-00825-6

๑) Springer Nature Limited 2020 